Fetal Diagnosis and Therapy
Fetal Diagn Ther 2019;45:332-338

DOI: $10.1159 / 000489260$
Received: November 27, 2017

Accepted after revision: April 15, 2018

Published online: August 15, 2018

\title{
Evaluation of First-Trimester Physiological Midgut Herniation Using Three-Dimensional Ultrasound
}

\author{
Hein Bogers $^{a}$ Leonie Baken ${ }^{a}$ Titia E. Cohen-Overbeek ${ }^{a} \quad$ Anton H.J. Koning $^{b}$ \\ Sten P. Willemsen ${ }^{c}$ Peter J. van der Spek ${ }^{b}$ Niek Exalto $^{a}$ Eric A.P. Steegers ${ }^{a}$ \\ a Division of Obstetrics and Prenatal Medicine, Department of Obstetrics and Gynaecology, Erasmus MC, \\ University Medical Centre, Rotterdam, The Netherlands; ${ }^{\text {b}}$ Department of Bioinformatics, Erasmus MC, \\ University Medical Centre, Rotterdam, The Netherlands; ' ${ }^{\mathrm{C}}$ Department of Biostatistics, Erasmus MC, \\ University Medical Centre, Rotterdam, The Netherlands
}

\section{Keywords}

Three-dimensional imaging · Exomphalos · Midgut

herniation $\cdot$ Omphalocele $\cdot$ Virtual reality

\begin{abstract}
Introduction: The aim of this study was to investigate the development of midgut herniation in vivo using three-dimensional (3D) ultrasonographic volume and distance measurements and to create reference data for physiological midgut herniation in ongoing pregnancies in a tertiary hospital population. Materials and Methods: The transvaginal 3D ultrasound volumes of 112 women, seen weekly during the first trimester of pregnancy, were obtained and subsequently analysed in a virtual reality environment. The width of the umbilical cord insertion, the maximum diameter of the umbilical cord, and the volume of midgut herniation were measured from 6 until 13 weeks gestational age (GA). $\boldsymbol{R e}$ sults: All parameters had a positive relation with GA, crownrump length, and abdominal circumference. In approximately 1 of 10 volumes no midgut herniation could be ob-
\end{abstract}

served at 9 and 10 weeks GA. In $5.0 \%$ of the fetuses the presence of midgut herniation could still be visualised at 12 weeks GA. Conclusion: Reference charts for several dimensions of physiological midgut herniation were created. In the future, our data might be used as a reference in the first trimester for comparison in case of a suspected pathological omphalocele.

(c) 2018 The Author(s) Published by S. Karger AG, Basel

\section{Introduction}

A congenital omphalocele, defined as the presence of visceral contents in the umbilical cord, can be detected from 12 weeks gestational age (GA) onwards [1-3]. Before 12 weeks there is a temporary physiological midgut herniation. There is an increasing interest in detecting structural abnormalities in the first trimester of pregnancy $[4,5]$. Although a large congenital omphalocele can be detected before 12 weeks GA [6], the false-positive diagnosis rate may be as high as $32 \%$ during the first trimester 
[7] and small omphaloceles may disappear later in pregnancy [8]. Kagan et al. [9] even described spontaneous resolution at 20 weeks in $92.5 \%$ of first-trimester-diagnosed omphaloceles in euploid fetuses. Three-dimensional (3D) imaging may be helpful in confirming the diagnosis and in counselling patients [10].

Between 7 and 12 weeks GA the midgut herniates into the umbilical cord. This midgut herniation (sometimes called physiological omphalocele) is caused by a relatively rapid growth of the midgut, accompanied by a $270^{\circ}$ anticlockwise midgut rotation in the abdominal cavity. Return into the body is caused by rapid growth of the body or decrease in the length of the mesentery. Failure may result in a (pathological) congenital omphalocele. Since this does not explain the possible herniation of other organs such as the liver, Achiron et al. [11] differentiated between an omphalocele caused by failure to form the primitive umbilical ring and a failed return of the midgut from the umbilical cord. This would also explain the spontaneous resolution of small omphaloceles.

Herniation was already observed in an in vitro study at Carnegie stage 16 (about 7 weeks and 2 days GA), and at 11 weeks GA the herniated intestine was still present in half of the cases [2].

Technical developments in 3D ultrasound techniques, including $3 \mathrm{D}$ virtual reality (3D-VR), have resulted in progress in visualisation of the fetus and fetal volume measurements as well [12]. The aim of this study was to investigate the presence and size of midgut herniation in normally developing fetuses using 3D ultrasonographic volume and distance measurements and to develop reference charts for the dimensions of midgut herniation during organogenesis.

\section{Materials and Methods}

\section{Study Population and Samples}

This study was conducted in a periconception cohort study at a university hospital for which women were enrolled for first-trimester longitudinal 3D ultrasound measurements to evaluate fetal growth and development using new imaging techniques. Pregnant women who participated were enrolled between 6 and 8 weeks GA via the outpatient clinic of the Department of Obstetrics and Gynaecology and local midwifery practices. All women received once-weekly 3D ultrasound scans between $6^{+0}$ and $12^{+6}$ weeks GA. Only women less than 8 weeks pregnant with a singleton pregnancy entered the study for further analysis.

We selected from the cohort 141 women who had been enrolled in 2009 and from whom at least one volume had been obtained. Two pregnancies complicated with trisomy 21 and 3 with congenital anomalies were excluded. Three multiple pregnancies, 3 dropouts, 16 miscarriages, and 2 cases with intrauterine fetal de- mise had to be excluded as well, leaving 112 inclusions for analysis (online suppl. Fig. S1; for all online suppl. material, see www. karger.com/doi/10.1159/000489260).

Since exomphalos is a common feature of Edwards syndrome, after finishing the study we also looked at available ultrasound volumes of trisomy 18 cases that have been described before [13].

\section{Pregnancy Dating}

The GA was calculated according to the first day of the last menstrual period in case of a regular menstrual cycle of 28 days and adjusted for a longer or shorter cycle [14]. In case of a discrepancy in GA of $>7$ days between crown-rump length (CRL) and the last menstrual period or an unknown last menstrual period, the GA was calculated by using CRL according to Robinson and Fleming [15] at the end of the first trimester using the latest available measurement. In case of assisted reproductive technology, GA was determined by the date of oocyte retrieval plus 14 days in pregnancies conceived via in vitro fertilisation with or without intracytoplasmic sperm injection procedures, from the last menstrual period or insemination date plus 14 days in pregnancies conceived through intrauterine insemination, and from the day of embryo transfer plus 17 or 18 days in pregnancies originating from the transfer of cryopreserved embryos, depending on the number of days between oocyte retrieval and cryopreservation of the embryo.

\section{Materials}

The sonographic volumes were acquired using a Voluson E8 ultrasound machine (GE Medical Systems, Zipf, Austria) and obtained with a transvaginal scan (GE-probe RIC-6-12-D [4.5-11.9 $\mathrm{MHz}]$ ). With regard to the safety aspects of first-trimester ultrasound the thermal index and the mechanical index were kept $<1.0$, the examiners were qualified and experienced, and the aslow-as-reasonably-practicable principle was respected: the duration of the examination did not exceed $30 \mathrm{~min}$ and 3D images were stored for offline evaluation in order to reduce the exposure to ultrasound as much as possible [16]. The $3 \mathrm{D}$ volumes were converted to a Cartesian format and visualised in the BARCO I-Space (Barco N.V., Kortrijk, Belgium). This is a four-walled CAVETM like (Cave Automatic Virtual Environment) VR system [12]. The $\mathrm{V}$-Scope volume rendering application is used to create a "hologram" of the ultrasound volume that is being investigated, floating in space in front of the observer [17]. For our study the 3D volumes were resized (enlarged), rotated, and cropped when necessary, and grey-scale and opacity values were adjusted for optimal image quality.

\section{Measurements}

Using the 3D-VR wireless joystick, we first determined whether we could observe the midgut to protrude in the umbilical cord. If so, just above the level of the insertion we measured in a midsagittal plane the anteroposterior diameter of the abdomen (APD), perpendicular to the spine. In an axial plane we measured perpendicular to this diameter the transverse diameter of the abdomen (TD) in order to calculate the abdominal circumference (AC): AC $=\pi[0.75(\mathrm{APD}+\mathrm{TD})-\sqrt{ }(\mathrm{APD} \times \mathrm{TD}) / 4]$. Then, we measured the maximum width of the midgut herniation and the width of the cord insertion in an axial plane as well (Fig. 1). After these measurements we erased all voxels surrounding the midgut herniation. The volume of this free-floating midgut herniation could be determined semiautomatically by placing a seed point in the midgut 


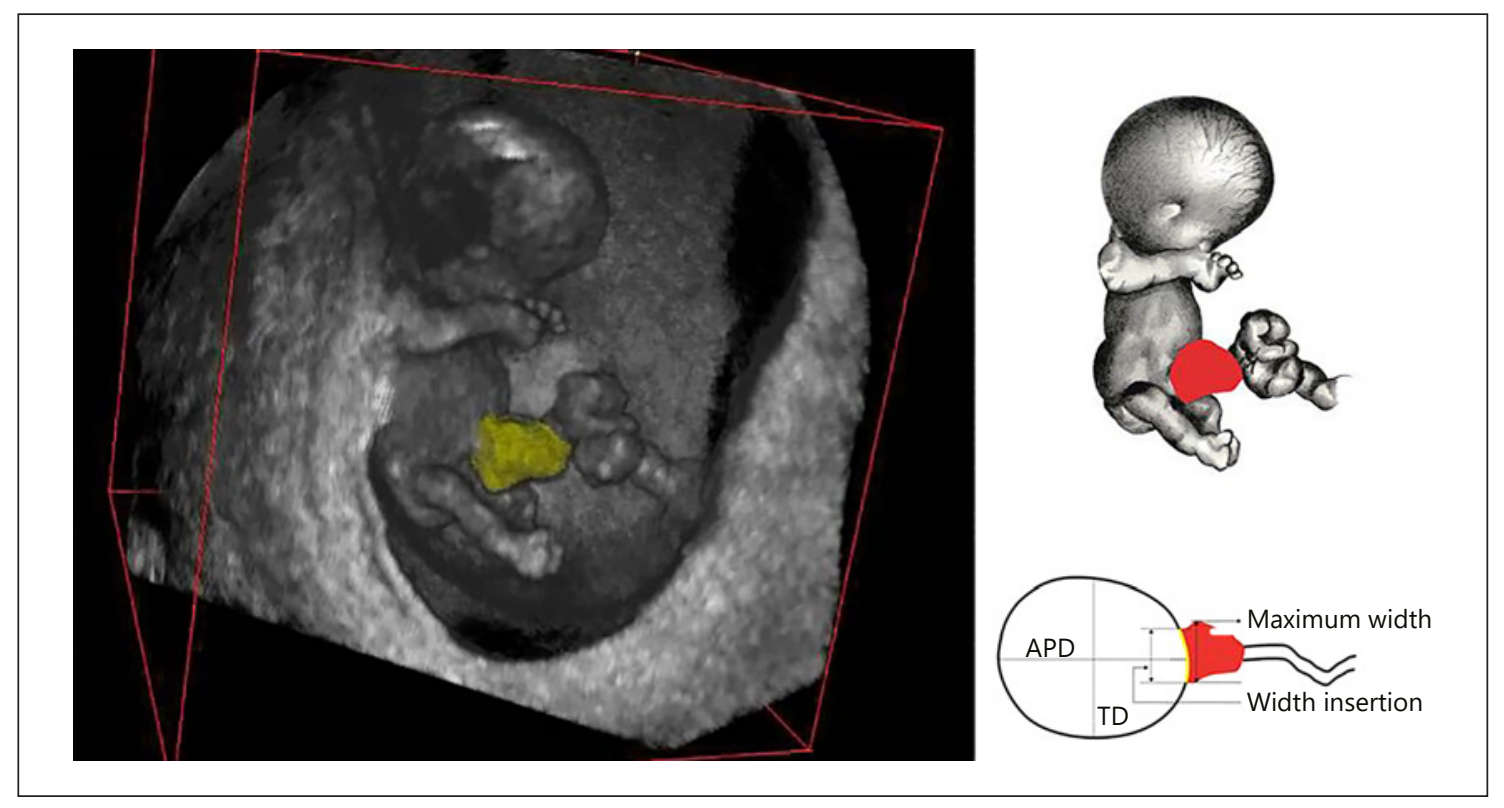

Fig. 1. Semiautomatic volume measurement of a free-floating midgut herniation in the BARCO I-Space (left) and schematic representation of measurements (right). APD, anteroposterior diameter of the abdomen; TD, transverse diameter of the abdomen.

Table 1. Absolute and relative numbers of absent and present midgut herniations and success percentages of measurements (measurement/number of volumes with discernible midgut herniation; \%) by gestational age (expressed in complete weeks)

\begin{tabular}{cccccccc}
\hline Week & Volume & $\begin{array}{l}\text { Midgut } \\
\text { herniation }-\end{array}$ & $\%$ & $\begin{array}{l}\text { Midgut } \\
\text { herniation }+\end{array}$ & $\%$ & Success rate & $\%$ \\
\hline 6 & 65 & 65 & 100.0 & 0 & 0.0 & - & - \\
7 & 101 & 91 & 90.1 & 10 & 9.9 & $3 / 10$ & 30.0 \\
8 & 106 & 47 & 44.3 & 59 & 55.7 & $29 / 59$ & 49.2 \\
9 & 107 & 10 & 9.3 & 97 & 90.7 & $62 / 97$ & 63.9 \\
10 & 110 & 12 & 10.9 & 98 & 89.1 & $67 / 98$ & 68.4 \\
11 & 109 & 73 & 67.0 & 36 & 33.0 & $17 / 36$ & 47.2 \\
12 & 101 & 96 & 95.0 & 5 & 5.0 & $3 / 5$ & 60.0 \\
\hline Total & 699 & 394 & 56.4 & 305 & 43.6 & $181 / 305$ & 59.3 \\
\hline
\end{tabular}

-, absent; +, present.

herniation and using the region growing segmentation algorithm implemented in the V-Scope application (Fig. 1).

All measurements were performed offline. The measurements of 31 fetuses were randomly repeated once by the same examiner (L.B.) and once by another examiner (H.B.) to study reproducibility by determining intra- and interobserver variability.

\section{Statistical Analysis}

We created reference curves for the maximum width of the midgut herniation and the width of the cord insertion as well as the volume of the midgut herniation. These parameters were plotted against GA, AC, and CRL. This was done using the generalised additive models for location scale and shape (GAMLSS) methodology using the eponymous $\mathrm{R}$ package [18]. It is assumed that the responses follow a (truncated) normal distribution after applying a Box-Cox transformation. The parameters of this distribution are modelled as a spline function of the independent variable.

Intra- and interobserver variability were depicted with BlandAltman plots indicating the bias (the systematic of mean difference between two measurements, with $95 \%$ confidence intervals) and the upper and lower limits of agreement (with $95 \%$ confidence intervals). The repeatability coefficient and intraclass correlation coefficients (two-way mixed, single measures, absolute agreement) of intra- and interobserver measurements were added. 

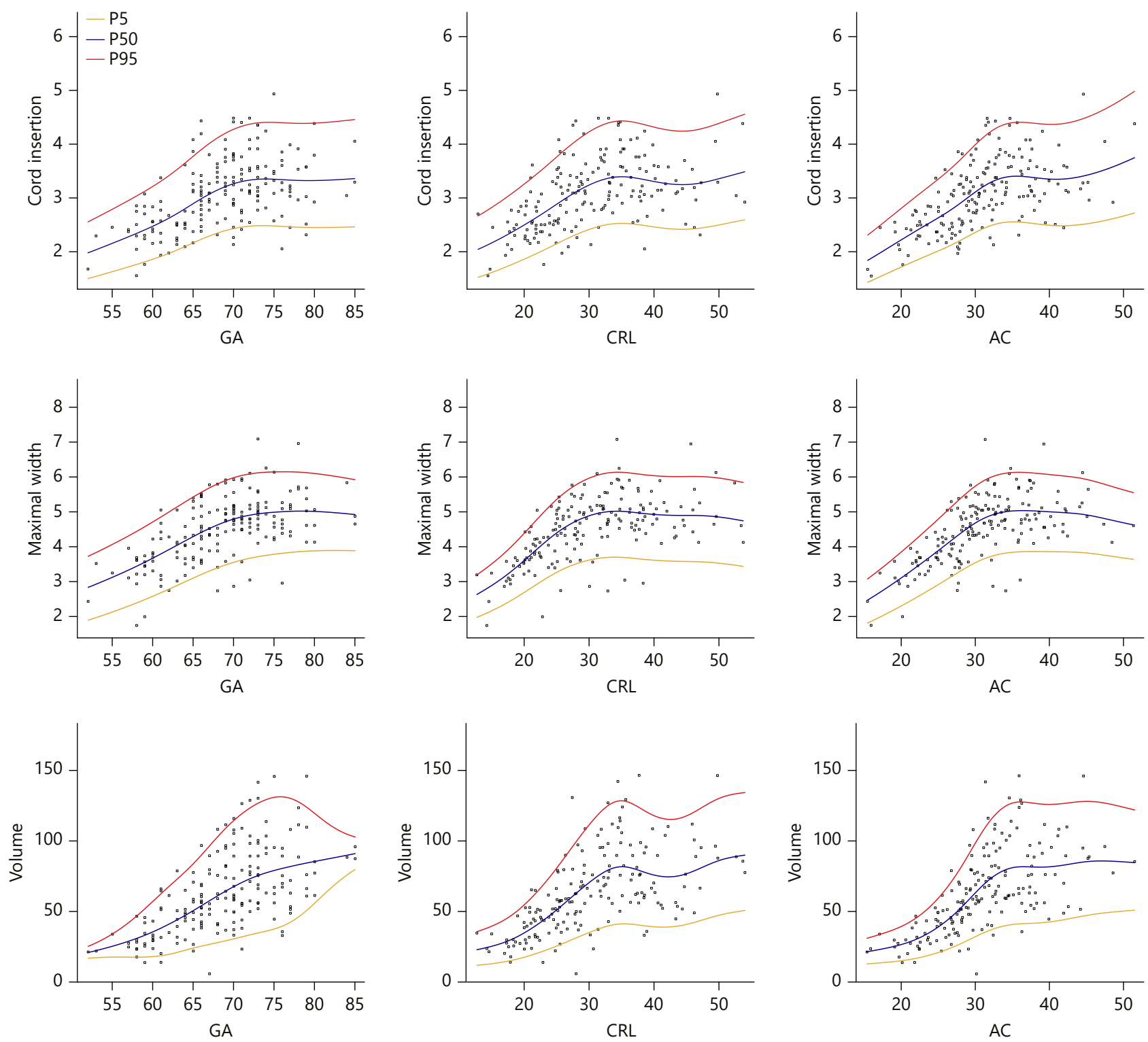

Fig. 2. Width of cord insertion ( $\mathrm{mm}$ ), maximum width of midgut herniation ( $\mathrm{mm})$, and volume of midgut herniation $\left(\mathrm{mm}^{3}\right)$ as a function of gestational age (GA; days), crown-rump length (CRL; $\mathrm{mm}$ ), and abdominal circumference (AC; mm).

\section{Results}

From the 112 pregnancies, 699 volumes were obtained for evaluation (mean 6.24, median 6, range 4-8 volumes per patient). Patient characteristics are presented in online supplementary Table S1. Of these 699 volumes, midgut herniation was present in 305 volumes. Measure- ments could be performed in 181 out of 305 volumes (59.3\%). Besides one fetus (with very low quality of all the acquired volumes), in all fetuses at some moment a herniation was seen. In our data set, in approximately 1 in 10 ultrasound volumes no midgut herniation could be observed at 9 and 10 weeks GA. The earliest and latest GA at which we were able to measure a midgut herniation was 
$7^{+3}$ and $12^{+1}$ weeks, respectively (Table 1 ). Reference values for the width of the cord insertion, the maximum width of the herniation, and the volume of the herniation are provided in online supplementary Tables S2, S3, and S4, respectively. In Figure 2 the width of the cord insertion is plotted against GA, CRL, and AC. Although there was a positive linear relation with all parameters, this effect was most prominent at early GA and smaller CRL and AC. The same results were obtained regarding the maximum width and the volume of the midgut herniation, plotted against the GA, CRL, and AC.

We found 6 cases of trisomy 18 between $12^{+0}$ weeks and $13^{+1}$ weeks, more or less immediately following the time period of study of our current paper $\left(7^{+3}-12^{+1}\right.$ weeks). Volume measurements in these 6 cases were much larger (median $182.6 \mathrm{~mm}^{3}$, range $71.4-995.6 \mathrm{~mm}^{3}$ ) as compared to our reference data (median $59.0 \mathrm{~mm}^{3}$, range $13.9-146.1 \mathrm{~mm}^{3}$ ).

The mean differences, the $95 \%$ limits of agreement, and the intraclass correlation coefficients of intra- and interobserver variability are shown in online supplementary Table S5. Intraclass correlation coefficients were all $>0.9$, indicating excellent reproducibility.

\section{Discussion}

We investigated the early fetal development of the umbilical cord insertion by means of 3D ultrasound. The midgut herniation could be measured reliably and reference curves were constructed for the volume and maximum width of the midgut herniation and the width of the umbilical cord insertion. Although we are not the first to have investigated midgut herniation by means of ultrasound, all other studies were performed in relatively few fetuses. Furthermore, most of them were done using $2 \mathrm{D}$ sonography $[1,3,6,19]$.

In 1987, Schmidt et al. [1] studied 14 women weekly between 7 and 12 weeks GA. The mean herniated mass was larger at 8 weeks GA (or 17-20 mm CRL) than at 9 weeks GA (or 23-26 CRL), which appears to be in contrast with our findings (Fig. 2). The authors, however, found a noticeable variation in size of the mass at the same GA.

Blaas et al. [3] investigated the development of several structures, including midgut herniation. Twentynine women were seen five times from 7 to 12 weeks GA to measure - among others - the thickness of the umbilical cord at the insertion and its thickness in a free loop. At 7 weeks GA, thickening of the cord containing a slight echogenic area was seen, progressing to a large hyperechogenic mass during 9 and 10 weeks GA. The thickness of the umbilical cord at the insertion appeared to be increased between 8 and 11 weeks GA compared with the thickness in a free loop. From $10^{+4}$ weeks the gut retracted, and from $11^{+5}$ weeks no herniation could be seen in any of the fetuses. In contrast, we still observed midgut herniation in a small minority of fetuses at 12 weeks GA.

Bowerman [19] investigated midgut herniation in 48 fetuses. From $11^{+2}$ weeks GA no herniated midgut could be observed. In all cases but one scanning was performed transabdominally. The significant gap between his and our findings might be caused by the fact that we used high-resolution transvaginal instead of transabdominal ultrasound, most likely resulting in higher image quality.

The circumference of midgut herniation was investigated weekly in 18 women by van Zalen-Sprock et al. [6] between 7 and 12 weeks GA. The circumference of the midgut herniation appeared to be greatest at 10 weeks $\mathrm{GA}$, with a maximum of $24.5 \mathrm{~mm}$ measured at the level of the insertion. Herniation could be observed in all fetuses at 10 weeks GA and in none of these at 12 weeks GA, which is in contrast with our findings (98 and 5\%, respectively). This may be due to the relatively small number of cases in comparison to the present study (18 vs. 110 and 101).

3D evaluation of midgut herniation has been investigated previously without performing measurements. Yonemoto et al. [20] and Hata et al. [21] compared - at 5-11 and 8-13 weeks GA - the visualisation rate of the midgut herniation, among others. They showed $3 \mathrm{D}$ examination to be non-inferior to $2 \mathrm{D}$ examination, although this was not scientifically tested. Soffers et al. [22], who investigated the rotation of the midgut in histological specimens, used another novel 3D visualisation technique in which serial sections of historical collections of embryos and fetuses were digitised using a scanning microscope. These images were subsequently converted and aligned to create a $3 \mathrm{D}$ reconstruction. The several loops of the midgut appear to develop in a hierarchal manner and to move independently of each other, facilitating phased return into the abdominal cavity. The authors state that the width of the hernia neck does not appear to determine the time window for intestinal return, which is supported by our finding that the width of the insertion does not increase after 10 weeks GA (Fig. 2).

To our best knowledge, we are the first to have performed volume measurements of herniation. Another 
strong point of our study is the number of volumes that could be analysed, although this was somewhat limited by the success rate of the measurements. This could be due to the fact that in our study only non-targeted 3D sweeps of the entire fetus were stored. Movement artefacts of the fetus could have impeded accurate visualisation. Furthermore, the parameters were measured offline on stored data. All these aspects contributed to the suboptimal success rate.

In 5 out of $101(5.0 \%)$ fetuses a midgut herniation could be observed at 12 weeks GA. Unfortunately we have only data available until 13 weeks GA. Further research in this period of gestation is necessary. The steeply decreasing number of fetuses with a midgut herniation from 11 weeks GA and the data from the literature however suggest a rapid disappearance after 12 weeks GA. In the careful postnatal follow-up of our study group no congenital omphaloceles were reported. The larger measurements in the trisomy 18 cases at least support the hypothesis that volume measurements may become a valuable diagnostic tool in diagnosing pathological omphaloceles at the end of the first trimester. One should remain cautious however as spontaneous resolution of omphaloceles has been described and a pathological omphalocele during this period can easily be mistaken for a physiological midgut herniation. Since in only 5\% of the fetuses at 12 weeks GA a midgut herniation was observed and the latest GA at which we could measure a herniation was $12^{+1}$ weeks, we advise to repeat the examination when a herniation is observed at 12 weeks GA, for this may be a pathological finding.

The proposed volume measurement technique cannot be done on conventional ultrasound machines with a $2 \mathrm{D}$ display, limiting its applicability. However, specialised $3 \mathrm{D}$ software is available to perform volume measurements on ultrasound machines or desktop computers. Also, a user-friendly 3D-VR desktop system has been developed for routine use of diagnostic 3D-VR ultrasound in the outpatient clinic, allowing precise length, volume, and angle measurements [23]. By means of this system the volume measurements can easily be performed.

\section{Conclusion}

We created reference data for midgut herniation in ongoing pregnancies. Our data may be used in the future for studies on the aetiology of abdominal wall defects and for comparison in fetuses in which a congenital, pathological omphalocele is suspected at the first-trimester ul- trasound scan. Since in only $5 \%$ of the fetuses at 12 weeks GA a midgut herniation was observed and the latest GA at which we could measure a herniation was $12^{+1}$ weeks, we advise to repeat the examination when a herniation is observed at 12 weeks GA, for this may be a pathological finding.

\section{Acknowledgment}

We would like to thank Hans Kneefel for help in creating Figure 1.

\section{Statement of Ethics}

All participants signed a written informed consent form and the local medical and ethical review committee approved the study protocol (METC 232.394/2003/177, METC 323.395/2003/178, MEC 2004-227).

\section{Disclosure Statement}

All authors declare not to have any conflict of interest.

\section{Funding Sources}

This research was solely funded by Erasmus MC, University Medical Centre, Rotterdam, The Netherlands. No external funding was obtained.

\section{Author Contributions}

H. Bogers and L. Baken were involved in data collection and measurements. The ultrasound measurements were supervised by N. Exalto, the BARCO I-Space measurements were supervised by A.H.J. Koning. S.P. Willemsen analysed the data. H. Bogers, T.E. Cohen-Overbeek, and N. Exalto wrote the first draft of the manuscript. E.A.P. Steegers was responsible for the infrastructure of the ultrasound measurements and the study population of the outpatient clinic. P.J. van der Spek was responsible for the infrastructure of the measurements in the BARCO I-Space. T.E. Cohen-Overbeek initiated and N. Exalto supervised all aspects of the study, including all revisions of the manuscript. All authors contributed to the preparation of the manuscript and approved the final version. 


\section{References}

1 Schmidt W, Yarkoni S, Crelin ES, Hobbins JC: Sonographic visualization of physiologic anterior abdominal wall hernia in the first trimester. Obstet Gynecol 1987;69:911-915.

2 Kim WK, Kim H, Ahn DH, Kim MH, Park HW: Timetable for intestinal rotation in staged human embryos and fetuses. Birth Defects Res A Clin Mol Teratol 2003;67:941945.

3 Blaas HG, Eik-Nes SH, Kiserud T, Hellevik LR: Early development of the abdominal wall, stomach and heart from 7 to 12 weeks of gestation: a longitudinal ultrasound study. Ultrasound Obstet Gynecol 1995;6:240-249.

4 Syngelaki A, Chelemen T, Dagklis T, Allan L, Nicolaides KH: Challenges in the diagnosis of fetal non-chromosomal abnormalities at 1113 weeks. Prenat Diagn 2011;31:90-102.

5 Chaoui R, Nicolaides KH: Detecting open spina bifida at the 11-13-week scan by assessing intracranial translucency and the posterior brain region: mid-sagittal or axial plane? Ultrasound Obstet Gynecol 2011;38:609-612.

6 van Zalen-Sprock RM, Vugt JM, van Geijn HP: First-trimester sonography of physiological midgut herniation and early diagnosis of omphalocele. Prenat Diagn 1997;17:511-518.

7 Lakasing L, Cicero S, Davenport M, Patel S, Nicolaides KH: Current outcome of antenatally diagnosed exomphalos: an 11 year review. J Pediatr Surg 2006;41:1403-1406.

8 Blazer S, Zimmer EZ, Gover A, Bronshtein M: Fetal omphalocele detected early in pregnancy: associated anomalies and outcomes. Radiology 2004;232:191-195.
9 Kagan KO, Staboulidou I, Syngelaki A, Cruz J, Nicolaides KH: The 11-13-week scan: diagnosis and outcome of holoprosencephaly, exomphalos and megacystis. Ultrasound $\mathrm{Ob}-$ stet Gynecol 2010;36:10-14.

10 Anandakumar C, Nuruddin Badruddin M, Chua TM, Wong YC, Chia D: First-trimester prenatal diagnosis of omphalocele using three-dimensional ultrasonography. Ultrasound Obstet Gynecol 2002;20:635-636.

11 Achiron R, Soriano D, Lipitz S, Mashiach S, Goldman B, Seidman DS: Fetal midgut herniation into the umbilical cord: improved definition of ventral abdominal anomaly with the use of transvaginal sonography. Ultrasound Obstet Gynecol 1995;6:256-260.

12 Rousian M, Hop WC, Koning AH, van der Spek PJ, Exalto N, Steegers EA: First trimester brain ventricle fluid and embryonic volumes measured by three-dimensional ultrasound with the use of I-Space virtual reality. Hum Reprod 2013;28:1181-1189.

13 Baken L, Benoit B, Koning AH, Willemsen SP, van der Spek PJ, Steegers-Theunissen RP, Steegers EA, Exalto N: First-trimester hand measurements in euploid and aneuploid human fetuses using virtual reality. Prenat Diagn 2014;34:961-969.

14 Steegers-Theunissen RP, Verheijden-Paulissen JJ, van Uitert EM, Wildhagen MF, Exalto N, Koning AH, Eggink AJ, Duvekot JJ, Laven JS, Tibboel D, Reiss I, Steegers EA: Cohort profile: the Rotterdam Periconceptional Cohort (Predict Study). Int J Epidemiol 2016;45: 374-381.

15 Robinson HP, Fleming JE: A critical evaluation of sonar "crown-rump length" measurements. Br J Obstet Gynaecol 1975;82:702710
16 Knez J, Day A, Jurkovic D: Ultrasound imaging in the management of bleeding and pain in early pregnancy. Best Pract Res Clin Obstet Gynaecol 2014;28:621-636.

17 Koning AH, Rousian M, Verwoerd-Dikkeboom CM, Goedknegt L, Steegers EA, van der Spek PJ: V-Scope: design and implementation of an immersive and desktop virtual reality volume visualization system. Stud Health Technol Inform 2009;142:136-138.

18 Stasinopoulos DM, Rigby RA: Generalized additive models for location scale and shape (GAMLSS) in R. J Stat Softw 2007;23:1-46.

19 Bowerman RA: Sonography of fetal midgut herniation: normal size criteria and correlation with crown-rump length. J Ultrasound Med 1993;12:251-254

20 Yonemoto H, Yoshida K, Kinoshita K, Takeuchi $\mathrm{H}$ : Embryological evaluation of surface features of human embryos and early fetuses by 3-D ultrasound. J Obstet Gynaecol Res 2002;28:211-216.

21 Hata T, Aoki S, Manabe A, Hata K, Miyazaki $\mathrm{K}$ : Three-dimensional ultrasonography in the first trimester of human pregnancy. Hum Reprod 1997;12:1800-1804.

22 Soffers JH, Hikspoors JP, Mekonen HK, Koehler SE, Lamers WH: The growth pattern of the human intestine and its mesentery. BMC Dev Biol 2015;15:31.

23 Baken L, van Gruting IM, Steegers EA, van der Spek PJ, Exalto N, Koning AH: Design and validation of a $3 \mathrm{D}$ virtual reality desktop system for sonographic length and volume measurements in early pregnancy evaluation. J Clin Ultrasound 2015;43:164-170. 